\title{
Heat transport through quantum Hall edge states: Tunneling versus capacitive coupling to reservoirs
}

\author{
Hugo Aita, ${ }^{1}$ Liliana Arrachea, ${ }^{2}$ Carlos Naón, ${ }^{1}$ and Eduardo Fradkin ${ }^{3}$ \\ ${ }^{1}$ Departamento de Física and IFLP, Facultad de Ciencias Exactas, Universidad Nacional de La Plata, cc 67, 1900, La Plata, Argentina \\ ${ }^{2}$ Departamento de Física and IFIBA, Facultad de Ciencias Exactas y Naturales, Universidad de Buenos Aires, \\ Pabellón 1, Ciudad Universitaria, 1428, Buenos Aires, Argentina \\ ${ }^{3}$ Department of Physics and Institute for Condensed Matter Theory, University of Illinois at Urbana-Champaign, \\ 1110 West Green Street, Urbana, Illinois 61801-3080, USA
}

(Received 24 May 2013; published 23 August 2013)

\begin{abstract}
We study the heat transport along an edge state of a two-dimensional electron gas in the quantum Hall regime, in contact to two reservoirs at different temperatures. We consider two exactly solvable models for the edge state coupled to the reservoirs. The first one corresponds to filling $v=1$ and tunneling coupling to the reservoirs. The second one corresponds to integer or fractional filling of the sequence $v=1 / m$ (with $m$ odd), and capacitive coupling to the reservoirs. In both cases, we solve the problem by means of nonequilibrium Green function formalism. We show that heat propagates chirally along the edge in the two setups. We identify two temperature regimes, defined by $\Delta$, the mean level spacing of the edge. At low temperatures, $T<\Delta$, finite size effects play an important role in heat transport, for both types of contacts. The nature of the contacts manifests itself in different power laws for the thermal conductance as a function of the temperature. For capacitive couplings, a highly nonuniversal behavior takes place, through a prefactor that depends on the length of the edge as well as on the coupling strengths and the filling fraction. For larger temperatures, $T>\Delta$, finite-size effects become irrelevant, but the heat transport strongly depends on the strength of the edge-reservoir interactions, in both cases. The thermal conductance for tunneling coupling grows linearly with $T$, whereas for the capacitive case, it saturates to a value that depends on the coupling strengths and the filling factors of the edge and the contacts.
\end{abstract}

DOI: 10.1103/PhysRevB.88.085122

PACS number(s): 72.10.Bg, 73.43.Jn, 73.23.Ad

\section{INTRODUCTION}

One of the most remarkable properties of the quantum Hall effect (QHE) is the existence of topologically protected chiral edge states. ${ }^{1}$ Originally unveiled by Laughlin ${ }^{2}$ and Halperin, ${ }^{3}$ the remarkable stability of these states is a consequence of the peculiar chiral $^{3,4}$ and topological ${ }^{5}$ nature of the quantum Hall effect. After the works of Wen ${ }^{6,7}$ and Kane and Fisher, ${ }^{8}$ these states are viewed as realizations of a chiral Luttinger liquid, which is amenable to be investigated by means of transport experiments.

The structure of the edge states reveals fundamental properties of the quantum Hall state. At filling fraction $v=1$, it consists of a single state located at the edge of the sample where electrons propagate chirally. Fractional quantum Hall states generally have a more complex structure of edge states, with one or more edge states. In general, each edge state has a chirality (which can be different from the other edge states) and its excitations carry nontrivial quantum numbers such as (generally fractional) charge as well as spin. Some edge states do not carry charge (or spin) excitations at all and are thus neutral. Thus edge states of fractional quantum Hall states contribute in nontrivial ways to the charge (and possibly spin) transport in the system. In addition, the edge states carry energy and hence contribute to the thermal transport. These interesting features have been recently investigated in systems in the integer and fractional Hall by means of different thermometry techniques. ${ }^{9-14}$ In this paper, we will focus on the energy (heat) transport properties of the edge states of the simplest fractional quantum Hall states, the Laughlin states.
Experimental evidence of the chiral propagation of the heat along an edge state in a GaAs/AlAs heterostructure with a two-dimensional electron gas in the integer quantum Hall regime has been presented in Ref. 9. The experiment was performed in the quantum Hall regime with filling $v=1$ locally heated by injecting an ac current from a source reservoir. These experimental features can be captured by a simple one-dimensional model of noninteracting chiral fermions connected to reservoirs through tunneling couplings. ${ }^{15} \mathrm{It}$ can be argued within that model that an analogous chiral propagation of the heat is expected if a stationary temperature gradient is applied between source and drain reservoirs, instead of heating with an ac current. Recent improvements in the technology of the edge state manipulations also enable the possibility of capacitive couplings. ${ }^{16}$ These results show that tunneling and capacitive couplings can be controlled, separately, if the sizes of contacts are selected appropriately. According to measurements in a quantum Hall Fabry-Perot interferometer, ${ }^{17}$ for an $18-\mu \mathrm{m}^{2}$ device, Coulomb effects are not significant. The same study shows zero-bias oscillations in a $2-\mu \mathrm{m}^{2}$ device of similar design, indicating (as expected) an increasing importance of charging effects in smaller samples.

While for the case of tunneling coupling the heat current is accompanied with a particle current, for capacitive couplings, the energy currents are isolated from the particle flow. This feature is interesting since it opens the possibility for the study of energy and charge propagation separately. Although in any realistic setup, the tunneling coupling is always present, and it is always more relevant than the capacitive coupling, the 
experiments of Ref. 16 show that it is possible to have a wide enough range of temperatures and voltages in which the tunneling coupling can be made small enough to be neglected.

Charge transport by tunneling coupling into the edge states has been the subject of many theoretical works. A limited list of papers on the topic is given by Refs. 1, 8, 18, and 19. Heat transport along edge states has been considered in a smaller number of studies. ${ }^{20-22}$ In addition to the work by Kane and Fisher, ${ }^{23}$ we can mention Ref. 15, which focuses on an ac driven edge corresponding to a filling $v=1$ and follows the experimental work by Granger et al. ${ }^{9}$ Another important recent work is Ref. 24, which is devoted to analyze thermoelectric effects between edge states through a coupled quantum dot in a quantum Hall bar with fillings $v=5 / 2$ and $2 / 3$.

The aim of the present work is to analyze heat transport induced by a temperature gradient applied at reservoirs that are capacitively coupled to an edge state of a quantum Hall state with filling $v$. We will consider the cases of an integer quantum Hall state, with $v=1$, and of general Laughlin fractional quantum Hall states, with filling fraction $v=1 / m$, with $m$ odd. We solve this problem exactly. For the particular case of filling $v=1$, we compare with the behavior of the heat transport induced by a temperature gradient at reservoirs connected to the edge by tunneling at point contacts, which is also an exactly solvable problem. The more general case, which involves tunneling at point contacts, is not exactly solvable and will be discussed elsewhere. In all the cases, we focus on two properties: (1) the thermal conductance of the edge and (2) the behavior of the local temperature along the nonequilibrium edge. The latter is defined by recourse to a "thermometer," which is realized by a third reservoir, which is very weakly coupled to the edge. The temperature of this reservoir is such that the heat flow through the contact vanishes. In this paper, we show that there is a different qualitative behavior of the heat conductance for tunneling and capacitive couplings. The behavior of the local temperature is, however, very similar in both cases. The local temperature displays a profile with discontinuities at the contacts to the reservoirs, indicating that the edge tends to thermalize with the closest upstream reservoir.

The paper is organized as follows. In Sec. II, we introduce two models to be exactly solved and define the heat currents in both cases. In Sec. III, we discuss the energy balance along the devices and give explicit formal expressions for heat currents in terms of correlators. In Sec. IV, we present the calculations of the heat transport using a Keldysh nonequilibrium Green function formalism. In Sec. V, we present results for the behavior of the local temperature along the edge as well as the thermal conductance. Section VI is devoted to summary and conclusions. Finally, in Appendices A and B, we gather some details of the calculations.

\section{MODELS}

The full system under consideration is sketched in Fig. 1. It is described by the following Hamiltonian:

$$
H=H_{\text {edge }}+\sum_{\alpha=1}^{M}\left(H_{\alpha}+H_{c, \alpha}\right) .
$$

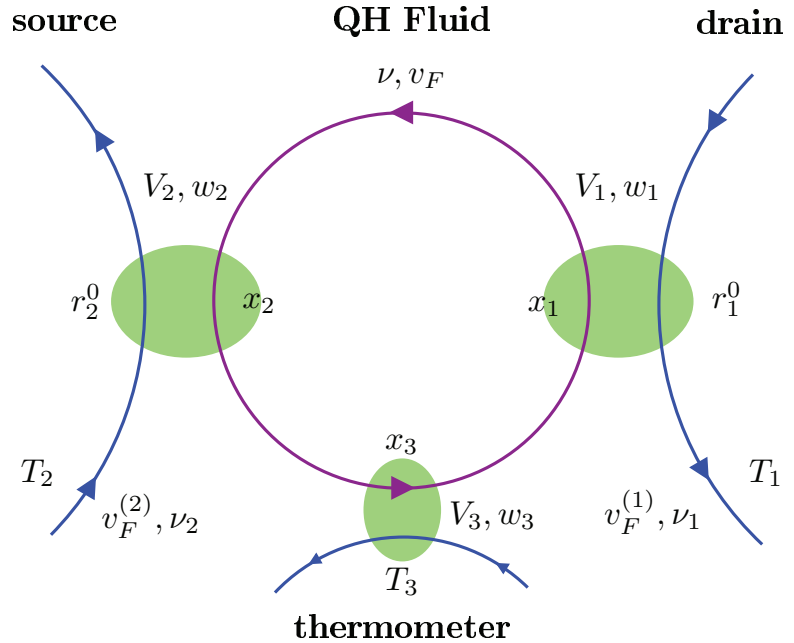

FIG. 1. (Color online) Sketch of the studied setup of a fractional (Laughlin) quantum Hall fluid in contact with a source and a drain and with a thermometer. The fractional quantum Hall edge state is represented by a ring. Two reservoirs, drain and source, with temperatures $T_{1}<T_{2}$, are connected to the ring at positions $x_{1}$ and $x_{2}$, either through contacts that allow tunneling of particles with couplings strengths $w_{1}, w_{2}$, or through capacitive couplings with strengths $V_{1}, V_{2}$ where only an energy current can flow through the leads. A third reservoir is weakly connected at $x_{3}$ in order to sense the local temperature $T_{3}$. For tunneling contacts, the only exactly solvable case corresponds to filling $v=1$. For capacitive coupling, any filling $v$ can be exactly treated.

The edge states of a quantum Hall fluid are represented by a one-dimensional (1D) system, a ring of circumference $L$, along which chiral fermions circulate with velocity $v_{F}$. The edge of the fluid is coupled to $M$ reservoirs (the leads) of infinite length, which are being represented by a set of "edge states" with fillings $v=1$, for tunneling coupling, or belonging to the Laughlin series, in the capacitive case. We will focus, in particular, on the configuration sketched in Fig. 1, with $M=3$ reservoirs. The drain $(\alpha=1)$ and source $(\alpha=2)$ reservoirs are at temperatures $T_{1}$ and $T_{2}$, respectively, with $T_{2}>T_{1}$. The third reservoir corresponds to a thermometer which senses the local temperature $T_{3}$. The latter is defined from the condition of local thermal equilibrium, implying a vanishing heat flow between this reservoir and the edge. We work in units where $\hbar=k_{B}=e=1$, but we will restore this universal constants in the discussion of the results.

\section{A. Tunneling coupling}

In this case, we focus on a quantum Hall state with integer filling $v=1$, which is the only exactly solvable case for the present geometry and under the effects of a temperature gradient. The Hamiltonian is

$$
\begin{aligned}
H_{\text {edge }} & =-i v_{F} \int_{0}^{L} d x: \Psi^{\dagger}(x) \partial_{x} \Psi(x): \\
& =\sum_{p} v_{F} p c_{p}^{\dagger} c_{p},
\end{aligned}
$$

where $p=2 n \pi / L$, with $n$ an integer. A large upper (UV) momentum cutoff $\Lambda=D / v$ (where $D$ is the bandwidth of the 
edge states) will be assumed. The bandwidth $D$ will also be assumed to be small compared to the gap that separates the Landau levels.

We represent the reservoirs by systems of $1 \mathrm{D}$ chiral fermions of length $L_{\alpha}$, which we will assume to be thermodynamically large, $L_{\alpha} \rightarrow \infty$. The corresponding Hamiltonian $H_{\alpha}$ for each of these systems reads

$$
H_{\alpha}=-i v_{F}^{\alpha} \int_{0}^{L_{\alpha}} d r_{\alpha} \Psi^{\dagger}\left(r_{\alpha}\right) \partial_{r_{\alpha}} \Psi\left(r_{\alpha}\right)
$$

The source and drain reservoirs, as well as the thermometer, have the same chemical potential $\mu$.

The contacts are described by the Hamiltonians

$$
H_{c, \alpha}=w_{\alpha} \sum_{\sigma}\left[\Psi_{\sigma}^{\dagger}\left(x_{\alpha}\right) \Psi_{\sigma}\left(r_{\alpha}^{0}\right)+\text { H.c. }\right],
$$

where $x_{\alpha}$ and $r_{\alpha}^{0}$ are, respectively, the positions of the ring and the reservoir at which the contact is established. We assume that the tunneling parameter $w_{3}$ between the ring and the thermometer is so weak that it introduces negligible dephasing in the particle propagation along the ring.

\section{B. Capacitive coupling}

In this section, we define the model corresponding to a capacitive coupling between the edge and the reservoirs. Assuming a local coupling, this corresponds to considering the Hamiltonian of Eq. (1) with the terms $H_{c, \alpha}=$ $V_{\alpha} \Psi^{\dagger}\left(x_{\alpha}\right) \Psi\left(x_{\alpha}\right) \Psi^{\dagger}\left(r_{\alpha}^{0}\right) \Psi\left(r_{\alpha}^{0}\right)$, where $r_{\alpha}^{0}$ and $x_{\alpha}$ are coordinates of the reservoir and the edge, respectively. As it is well known, this type of quartic interactions can be more easily handled by adopting a bosonic representation of the edge states. ${ }^{25}$ In that language, the fermionic density becomes proportional to the spatial derivative of a free chiral bosonic field $\phi(x)$, which represents a quantum fluctuation propagating along the edge of the quantum Hall fluid. Since the bulk of the quantum Hall fluid is gapped (and hence incompressible), the edge of the fluid can be regarded as a ring of finite length $L$ of noninteracting chiral bosons (with a fixed "compactification radius" determined by the filling fraction of the bulk quantum Hall fluid, see Ref. 1) capacitively coupled to reservoirs at different temperatures. The reservoirs are also described by 1D chiral bosons of infinite length, with fillings $v_{\alpha}$.

The total Hamiltonian has the structure of Eq. (1). In the bosonized language, the Hamiltonian for the edge is given by

$$
H_{\text {edge }}=\frac{v_{F}}{4 \pi v} \int_{0}^{L} d x:\left(\partial_{x} \phi(x)\right)^{2}:+\frac{\pi}{L} \hat{N}(\hat{N}+1),
$$

where $\hat{N}$ is the number operator corresponding to the original fermionic system (see Ref. 26 for details) and $v$ is the filling fraction. The present case can be solved for a quantum Hall state with a filling fraction $v$, which can be integer as well as fractional with the law $v=1 / m$, with $m$ odd.

The Hamiltonians for the reservoirs read

$$
H_{\alpha}=\frac{v_{F}^{\alpha}}{4 \pi v_{\alpha}} \int_{0}^{L_{\alpha}} d r_{\alpha}:\left(\partial_{r_{\alpha}} \phi\left(r_{\alpha}\right)\right)^{2}: .
$$

As in the tunneling case, we will consider the leads to be infinitely long, $L_{\alpha} \rightarrow \infty$. The contact between the central system and the two reservoirs is

$$
H_{c, \alpha}=\left.\left.V_{\alpha} \partial_{r_{\alpha}} \phi\left(r_{\alpha}\right)\right|_{r_{\alpha}=r_{\alpha}^{0}} \partial_{x} \phi(x)\right|_{x=x_{\alpha}},
$$

where $x_{\alpha}$ and $r_{\alpha}^{0}$ are the points on the ring and the reservoir, respectively, that intervene in the coupling.

The chiral Bose fields $\phi(x)$ and $\phi\left(r_{\alpha}\right)$ satisfy the equal-time commutation relations:

$$
\begin{gathered}
{\left[\phi\left(r_{\alpha}\right), \phi(x)\right]=0,} \\
{\left[\phi(x), \phi\left(x^{\prime}\right)\right]=-i \pi \nu \operatorname{sgn}\left(x-x^{\prime}\right),} \\
{\left[\phi\left(r_{\alpha}\right), \phi\left(r_{\alpha}^{\prime}\right)\right]=-i \pi v_{\alpha} \operatorname{sgn}\left(r_{\alpha}-r_{\alpha}^{\prime}\right) .}
\end{gathered}
$$

\section{ENERGY BALANCE AND HEAT CURRENT}

Our aim is to evaluate the heat current flowing through the contacts between the edge state and a given reservoir $\alpha$. To this end, we analyze the time dependence of the energy stored in the reservoir. In the case of the tunneling coupling, we consider

$$
\dot{Q}_{\alpha}=\dot{E}_{\alpha}-\mu \dot{N}_{\alpha}=-i\left\langle\left[H_{\alpha}-\mu N_{\alpha}, H_{c, \alpha}\right]\right\rangle=J_{\alpha}^{Q, \mathrm{t}},
$$

where $E_{\alpha}$ and $N_{\alpha}$ are, respectively, the energy and the charge stored in the reservoir $\alpha$. In order to relate energy flow to heat flow we subtracted the convective component $\mu \dot{N}_{\alpha}$. The result is

$$
J_{\alpha}^{Q, \mathrm{t}}=-2 \operatorname{Re}\left[\int \frac{d p_{\alpha}}{2 \pi} w_{p_{\alpha}}\left(\varepsilon_{p_{\alpha}}-\mu\right) \tilde{G}^{<}\left(x_{\alpha}, p_{\alpha} ; t, t\right)\right],
$$

where $\varepsilon_{p_{\alpha}}=v_{F}^{\alpha} p_{\alpha}$ and $w_{p_{\alpha}}=w_{\alpha} e^{-i p_{\alpha} r_{\alpha}^{0}} / \sqrt{L_{\alpha}}$. The lesser Green function is

$$
\tilde{G}^{<}\left(x_{\alpha}, p_{\alpha} ; t, t^{\prime}\right)=i\left\langle c_{p_{\alpha}}^{\dagger}\left(t^{\prime}\right) \Psi\left(x_{\alpha}, t\right)\right\rangle .
$$

In the case of the capacitive coupling, there is no particle flow. Thus the energy flow is equivalent to the heat flow:

$$
\dot{Q}_{\alpha}=\dot{E}_{\alpha}=-i\left\langle\left[H_{\alpha}, H_{c, \alpha}\right]\right\rangle=J_{\alpha}^{Q, \mathrm{c}} .
$$

The calculation yields

$$
J_{\alpha}^{Q, \mathrm{c}}=\left.i V_{\alpha} v_{F}^{\alpha} \partial_{x} \partial_{r_{\alpha}}^{2} \tilde{D}^{<}\left(x, r_{\alpha} ; t, t\right)\right|_{x=x_{\alpha}, r=r_{\alpha}^{0}},
$$

with the lesser function defined as

$$
\tilde{D}^{<}\left(x, r_{\alpha} ; t, t^{\prime}\right)=i\left\langle\phi\left(r_{\alpha}\right)\left(t^{\prime}\right) \phi(x, t)\right\rangle .
$$

Notice that $\left[\hat{N}, \partial_{x} \phi(x)\right]=0$, thus the last term of Eq. (5) does not contribute to the heat current.

\section{METHODOLOGY: NONEQUILIBRIUM GREEN FUNCTIONS}

\section{A. Tunneling coupling}

In order to compute the current, we must evaluate the lesser Green function given in Eq. (13). To this end, we define the retarded Green function

$$
G^{R}\left(x, x^{\prime} ; t, t^{\prime}\right)=-i \Theta\left(t-t^{\prime}\right)\left\langle\left\{\Psi(x, t), \Psi^{\dagger}\left(x^{\prime}, t^{\prime}\right)\right\}\right\rangle,
$$

where $x, x^{\prime}$ are coordinates on the ring. This is a rather standard procedure, which we summarize for completeness in Appendix A. The lesser Green function entering the expression 
of the current of Eq. (12) can be calculated from Eq. (A13). The result is

$$
\begin{aligned}
J_{\alpha}^{Q, \mathrm{t}}= & -\int_{-\infty}^{+\infty} \frac{d \omega}{2 \pi}(\omega-\mu) \Gamma_{\alpha}^{\mathrm{t}}(\omega)\left\{2 \operatorname{Im}\left[G^{R}\left(x_{\alpha}, x_{\alpha} ; \omega\right) f_{\alpha}(\omega)\right]\right. \\
& \left.+\sum_{\beta=1}^{M}\left|G^{R}\left(x_{\alpha}, x_{\beta} ; \omega\right)\right|^{2} \Gamma_{\beta}^{\mathrm{t}}(\omega) f_{\beta}(\omega)\right\}
\end{aligned}
$$

where the function $G^{R}\left(x_{\alpha}, x_{\beta} ; \omega\right)$ is obtained from the second equation of the set of Eq. (A8) and $f_{\alpha}(\omega)$ is the FermiDirac function, which depends on the chemical potential and temperature of the reservoir $\alpha$. The hybridization function $\Gamma_{\alpha}^{\mathrm{t}}(\omega)$, defined in Eq. (A11), depends on the density of states of the reservoir, which in our case, is a constant within the bandwidth characterized by an energy cutoff $\Lambda$, and the square of the tunneling amplitude $\left|w_{\alpha}\right|^{2}$ between the edge and the reservoir.

An alternative representation for this current is obtained by substituting the identity of Eq. (A13) into Eq. (18). The resulting expression reads

$$
J_{\alpha}^{Q, \mathrm{t}}=\sum_{\beta=1}^{M} \int_{-\infty}^{+\infty} \frac{d \omega}{2 \pi}(\omega-\mu) \mathcal{T}_{\alpha, \beta}^{\mathrm{t}}(\omega)\left[f_{\alpha}(\omega)-f_{\beta}(\omega)\right],
$$

which has the familiar form of a Landauer-Büttiker formula. The heat current resulting from a difference of temperatures imposed at the reservoirs is expressed in terms of the corresponding difference of Fermi functions times the amount of heat transferred by the tunneling of particles, $\omega-\mu$, times the transmission function, which quantifies the transparency of the system in contact to the reservoirs. The latter function in our case reads

$$
\mathcal{T}_{\alpha, \beta}^{\mathrm{t}}(\omega)=\Gamma_{\alpha}^{\mathrm{t}}(\omega)\left|G^{R}\left(x_{\alpha}, x_{\beta} ; \omega\right)\right|^{2} \Gamma_{\beta}^{\mathrm{t}}(\omega),
$$

which depends on the Green function of the coupled edge and the hybridization functions of the coupled reservoirs.

A typical plot for the transmission function $\mathcal{T}_{1,2}^{\mathrm{t}}(\omega)$ of a two terminal setup is shown in Fig. 2(a). It is evaluated by solving the set of two coupled equations defined by Eq. (A8) for $M=2$ reservoirs and $x^{\prime}=x_{\alpha}$, with $\alpha=1,2$. The result is a sequence of resonances which define Lorentzian peaks at the positions $\varepsilon_{k_{n}}=v_{F} 2 \pi n / L$ of the energies of the isolated edge. The hybridization to the reservoirs generate finite lifetime of the electrons occupying those states, which is accounted by the width $\propto \Gamma_{\alpha}^{\mathrm{t}}$ of the peaks of the transmission function. For the present model of reservoirs, the widths as well as the heights of the resonant peaks, are constant.

\section{B. Capacitive coupling}

In this case, the evaluation of the heat current of Eq. (15) requires the computation of the lesser function which is given by Eq. (16). We define the retarded Green function

$$
D^{R}\left(x, x^{\prime} ; t, t^{\prime}\right)=-i \Theta\left(t-t^{\prime}\right)\left\langle\left[\phi(x, t), \phi\left(x^{\prime}, t^{\prime}\right)\right]\right\rangle .
$$

In Appendix B, we present the calculation of the corresponding Dyson equations. Upon substituting Eq. (B19) into Eq. (15),

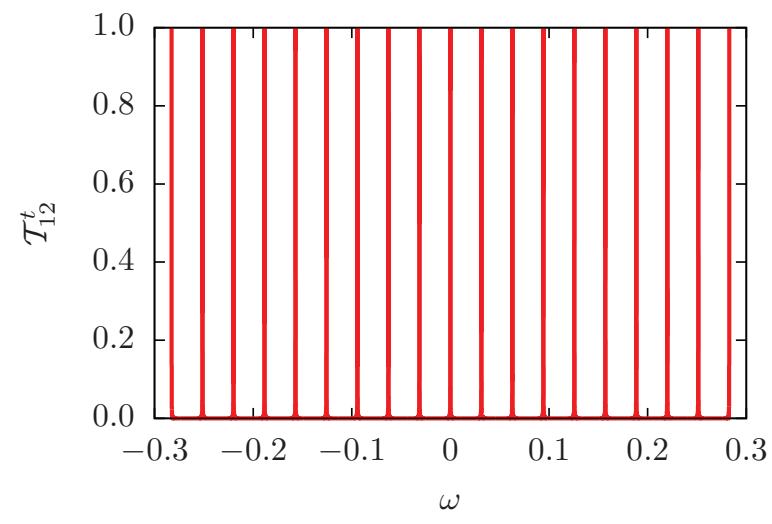

(a) Tunneling coupling

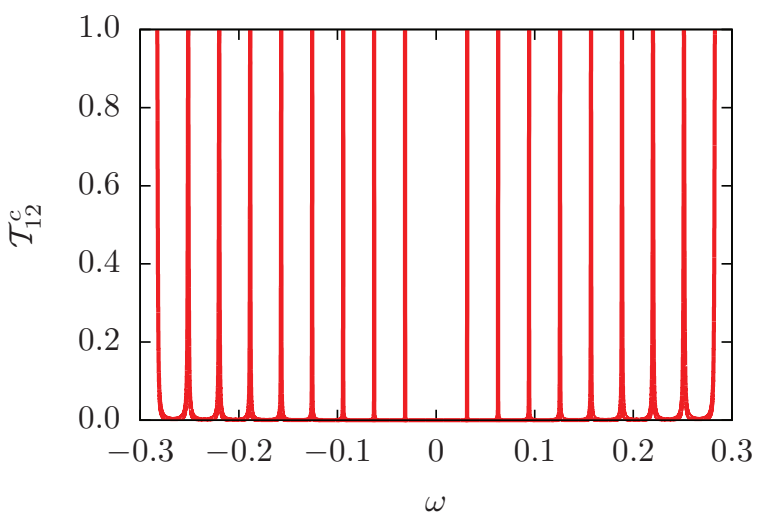

(b) Capacitive coupling

FIG. 2. (Color online) (Top) Transmission function $\mathcal{T}_{12} \mathrm{t}$ as function of $\omega$ for a tunneling coupling between the edge and two reservoirs with amplitudes $w_{1}=w_{2}=0.1$. (Bottom) Transmission function $\mathcal{T}_{12} \mathrm{c}$ as function of $\omega$ for a capacitive coupling between the edge and two reservoirs with amplitudes $V_{1}=V_{2}=0.2$. The remaining parameters, common to the two cases, are $v_{F}=1$ and $L=200$, $x_{1}=0$ and $x_{2}=100$. All energies are expressed in natural units $(\hbar=1)$.

we get the explicit expression

$$
\begin{aligned}
J_{\alpha}^{Q, \mathrm{c}}= & -\frac{1}{2} \int_{-\infty}^{+\infty} \omega \Gamma_{\alpha}^{\mathrm{c}}(\omega)\left\{2 \operatorname{Im}\left[\mathcal{D}^{R}\left(x_{\alpha}, x_{\alpha} ; \omega\right)\right] n_{\alpha}(\omega)\right. \\
& \left.+\sum_{\beta=1}^{M}\left|\mathcal{D}^{R}\left(x_{\alpha}, x_{\beta} ; \omega\right)\right|^{2} \Gamma_{\beta}^{\mathrm{c}}(\omega) n_{\beta}(\omega)\right\}
\end{aligned}
$$

where the retarded Green function $\mathcal{D}^{R}\left(x, x^{\prime} ; \omega\right)$ is defined in Eq. (B9). Using the identity given in Eq. (B14), the heat current can be expressed as

$$
J_{\alpha}^{Q, \mathrm{c}}=\sum_{\beta=1}^{M} \int_{-\infty}^{+\infty} \frac{d \omega}{2 \pi} \omega \mathcal{T}_{\alpha, \beta}^{\mathrm{c}}(\omega)\left[n_{\alpha}(\omega)-n_{\beta}(\omega)\right],
$$

where we have defined the transmission function

$$
\mathcal{T}_{\alpha, \beta}^{\mathrm{c}}(\omega)=\Gamma_{\alpha}^{\mathrm{c}}(\omega)\left|\mathcal{D}^{R}\left(x_{\alpha}, x_{\beta} ; \omega\right)\right|^{2} \Gamma_{\beta}^{\mathrm{c}}(\omega) / 2 .
$$

This function has the same properties as its tunneling counterpart, $\mathcal{T}_{\alpha, \beta}^{\mathrm{t}}(\omega)$. In particular, it satisfies the symmetry

$$
\mathcal{T}_{\alpha, \beta}^{\mathrm{c}}(\omega)=\mathcal{T}_{\beta, \alpha}^{\mathrm{c}}(\omega), \forall \alpha, \beta,
$$

which implies the continuity of the heat current. 
The above expression for the heat current has the same structure as the corresponding one for tunneling contacts given in Eq. (19). The temperature difference imposed at the reservoirs enters in the present case in the Bose-Einstein functions instead of in the Fermi-Dirac ones. In the present case, there is no flow of particles. Thus the energy transferred $\omega$ is directly interpreted as heat. The transmission function depends on the amplitude of the capacitive couplings as well as on the spectral function of the bosonic reservoirs through the functions $\Gamma_{\alpha}^{\mathrm{c}}(\omega)$ defined in Eq. (B13).

A typical plot of the transmission function in a two-terminal configuration is shown in Fig. 2(b). In the present case, we must evaluate the linear set of two equations defined by Eq. (B8) with $M=2$ for $x^{\prime}=x_{\alpha}, \alpha=1,2$. As in the tunneling case, the result consists of a set of resonances with a spacing $\Delta \omega \sim 2 \pi v_{F} / L$ corresponding to the energies of the uncoupled ring. The coupling to the reservoirs introduces a finite lifetime, which determines the width $\propto|\omega| V_{\alpha}^{2}$ of the peaks of the transmission function. As in the case of the tunneling coupling, the height of these peaks achieves the maximum value, equal to one, at resonance. However, at low energies $|\omega|<$ $2 \pi v_{F} / L$, there is a strong suppression of the spectral weight. As we will discuss in the next section, this effect renders the transmission of heat vanishing small for low temperatures.

\section{RESULTS}

We now turn to the discussion of the behavior of the thermal transport through the edge state. We will analyze the thermal conductance and the local temperature sensed by a thermometer noninvasively coupled along the edge. We consider a two-terminal configuration with reservoirs at different temperatures, $T_{1}$ and $T_{2}$ connected, respectively, at $x=x_{1}$ and $x=x_{2}$.

\section{A. Thermal conductance}

We consider the source and drain reservoirs at slightly different temperatures $T_{1}=T$ and $T_{2}=T+\delta T$. The thermal conductance of the coupled edge state reads

$$
G_{\mathrm{th}}=\lim _{\delta T \rightarrow 0} \frac{J^{Q}(T+\delta T)-J^{Q}(T)}{\delta T},
$$

where $J^{Q}$ is the heat current flowing through the contacts between the edge and the reservoirs. Notice that it is the same for the two contacts because of the continuity of the energy and charge flows. In the forthcoming discussion presented within this section, it is convenient to restore the units in terms of the fundamental constants $h, e, k_{B}$.

\section{Tunneling coupling}

For tunneling coupling, we can recognize two different regimes: the mesoscopic case $\left(k_{B} T \ll \Delta\right)$ and the macroscopic one $(T \gg \Delta)$, where $\Delta=\hbar v_{F} 2 \pi / L$ is the level spacing of the edge. In the former, the conductance depends not only on $T$ but also on the chemical potential $\mu$. We distinguish two different situations depending on wether $\mu$ coincides with one of the energy levels of the edge (resonance) or it lies between two energy levels (off resonance). We hereafter focus on small $k_{B} T / \mu$ where we can resort to Sommerfeld expansion in Eq. (19) provided that we are in a regime where the transmission function is slowly varying with $\omega$. This expansion casts

$$
J^{Q, \mathrm{t}}=\left.\frac{k_{B}^{2} \pi^{2}}{3 h} \frac{d}{d \omega}\left[(\omega-\mu) \mathcal{T}_{2,1}^{\mathrm{t}}(\omega)\right]\right|_{\omega=\mu} T \delta T,
$$

which yields a linear behavior for the thermal conductance

$$
G_{\mathrm{th}}^{\mathrm{t}}(T)=\kappa_{0} \mathcal{T}_{2,1}^{\mathrm{t}}(\mu) T,
$$

where $\kappa_{0}=k_{B}^{2} \pi^{2} / 3 h$ is the universal thermal conductance quantum constant. As shown in Fig. 2(a), the transmission function has a structure with several peaks and can be approximated by the constant $\mathcal{T}_{2,1}^{\mathrm{t}}(\mu)$ only in two limits. The first one corresponds to a resonant $\mu$ and the ultra small range of temperatures $k_{B} T \ll \gamma$, being $\gamma$ the width of the peaks, where the transmission function is $\mathcal{T}_{2,1}^{\mathrm{t}}(\omega) \sim 1$. The second one corresponds to an off-resonant $\mu$ and also a small range $k_{B} T \ll \Delta$, where the transmission function is $\mathcal{T}_{2,1}^{\mathrm{t}}(\omega) \sim 0$.

In the macroscopic regime, $k_{B} T \gg \Delta$, the conductance also grows linearly with $T$ and does not depend on $\mu$,

$$
G_{\text {th }}^{\mathrm{t}}=f\left(\tilde{w}_{1}, \tilde{w}_{2}\right) \kappa_{0} T,
$$

where the function $f\left(\tilde{w}_{1}, \tilde{w}_{2}\right)$ has the form

$$
f\left(\tilde{w}_{1}, \tilde{w}_{2}\right)=\frac{4 \tilde{w}_{1}^{2} \tilde{w}_{2}^{2}}{\tilde{w}_{1}^{2}+\tilde{w}_{2}^{2}} \frac{1}{\left(1+\tilde{w}_{1}^{2} \tilde{w}_{2}^{2}\right)},
$$

where we have defined $\tilde{w}_{\alpha}=w_{\alpha} / 2 \hbar \sqrt{v_{F} v_{F}^{\alpha}}$. In Appendix C, we present an analytic derivation of this result. Interestingly, this implies that, in this regime, $G_{\text {th }}^{\mathrm{t}}$ has a nonmonotonic behavior as function of the ring-reservoir coupling strength, as shown in Fig. 3(a). A similar behavior has been previously found in Ref. 27 for the magnetization current in a XX spin- $1 / 2$ chain coupled to quantum reservoirs, and in steady state thermal current in an open XY spin-1/2 chain. ${ }^{28} \mathrm{We}$ have also verified that a similar behavior takes place for the thermal conductance of a tight-binding chain connected to one-dimensional electron reservoirs through a tunneling coupling with a mismatching. The fact that the conductance at a fixed $T$ grows as a function of the coupling to the reservoirs until a maximum value and then decreases for even larger couplings is a priori nonintuitive. Interestingly, it is a consequence of the coherent nature of the heat propagation. In fact, notice that the quantity $v_{\mathrm{t}}^{\alpha}=w_{\alpha} / 2 \hbar$ can be interpreted as the velocity with which the electrons travel through the tunneling coupling, while the quantity $\tilde{w}_{\alpha}^{2}=\left(v_{\mathrm{t}}^{\alpha} / v_{F}^{\alpha}\right)\left(v_{\mathrm{t}}^{\alpha} / v_{F}\right)$, entering in Eq. (30) is a measure of the velocity mismatch for the electron motion through the junction, the one within the reservoir and the one along the ring.

The behavior of Fig. 3(a) shows that a small thermal flow between the two reservoirs is expected for a high mismatching between these three velocities. This may occur for a very weak coupling $w_{\alpha}$ in which case the velocity of tunneling is much smaller than the velocities that the electrons have within the reservoirs and within the finite-size central edge. A similar effect is expected for a large $w_{\alpha}$, which corresponds to $v_{\mathrm{t}}^{\alpha} \gg v_{F}^{\alpha}, v_{\mathrm{t}}^{\alpha} \gg v_{F}$. In this case, the electrons jump through the contact at a much higher velocity than the one with which they propagate within the reservoirs and along the central ring, resulting in a poor net transmission from one reservoir to the other. It is important to notice that the function $f\left(\tilde{w}_{1}, \tilde{w}_{2}\right) \leqslant 1$. 


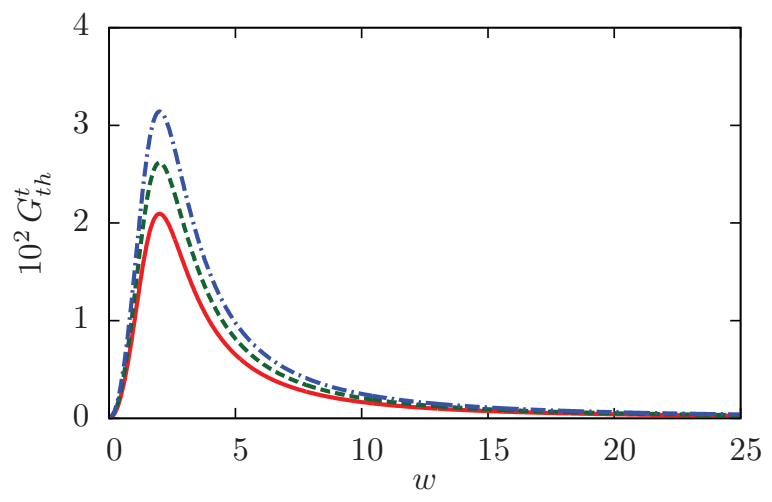

(a) Tunneling coupling

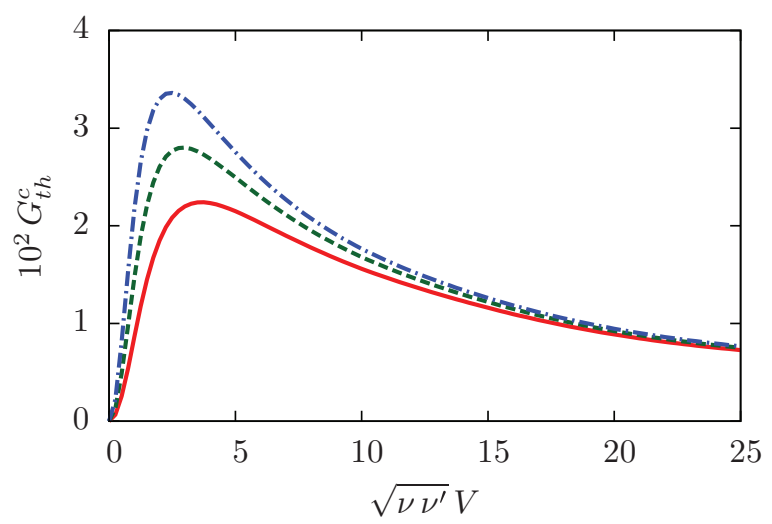

(b) Capacitive coupling

FIG. 3. (Color online) Behavior of the thermal conductance as a function of the coupling strength within the regime $k_{B} T \gg \Delta$. (Top) Tunneling thermal conductance $G_{\mathrm{th}}^{t}$, having set $w_{1}=w_{2}=w$, for different temperatures: $T=0.04$ (red, solid), $T=0.05$ (green, dashed), $T=0.06$ (blue, dot-dashed). (Bottom) Capacitive thermal conductance $G_{\mathrm{th}}^{c}$ as a function of $\sqrt{\nu \nu^{\prime}} V$ for different temperatures: $T=0.04$ (red, solid), 0.05 (green, dashed), and 0.06 (blue, dotdashed). We have set $V_{1}=V_{2}=V$ and $v_{1}=v_{2}=v^{\prime}$, and $v$ is the filling factor of the ring. All energies are expressed in natural units $(\hbar=1)$.

The thermal conductance is thus upper bounded by its ballistic value $\kappa_{0} T$ and satisfies the limit set in Ref. 31. An alternative heuristic derivation of this limit consists in requesting that the thermal conductance satisfies the uncertainty principle $\Delta E \tau \geqslant \hbar / 2$, where $\Delta E \sim k_{B} \delta T$ and $\tau=k_{B} T / J^{Q, \mathrm{t}}$. Using $J^{Q, \mathrm{t}}=G_{\mathrm{th}}^{\mathrm{t}} \delta T$, we get $G_{\mathrm{th}}^{\mathrm{t}} \leqslant 2 k_{B}^{2} T / \hbar$, which is approximately the exact upper bound.

The different regimes are illustrated in Fig. 4. The solid red plot corresponds to a resonant $\mu$ and we can distinguish a very narrow region close to $T=0$ where $G_{\mathrm{th}}^{\mathrm{t}}(T)$ grows linearly (see the inset of the figure). For $T>\gamma$, there is a structure related to the end of the peak, while for $T>\Delta$ it grows again linearly. In the other plots, corresponding to off-resonant $\mu$, it can be seen a vanishing conductance for $T<\left|\mu-\varepsilon_{c}\right|$, where $\varepsilon_{c}$ is the energy of the energy level of the edge that is the closest one to $\mu$. A structure (maximum) related to the existence of a neighboring peak is observed and for larger $T$ the conductance is again linear. This behavior is repeated as $\mu$ is varied through the sequence of peaks and valleys, as shown in the contour plot of Fig. 5.

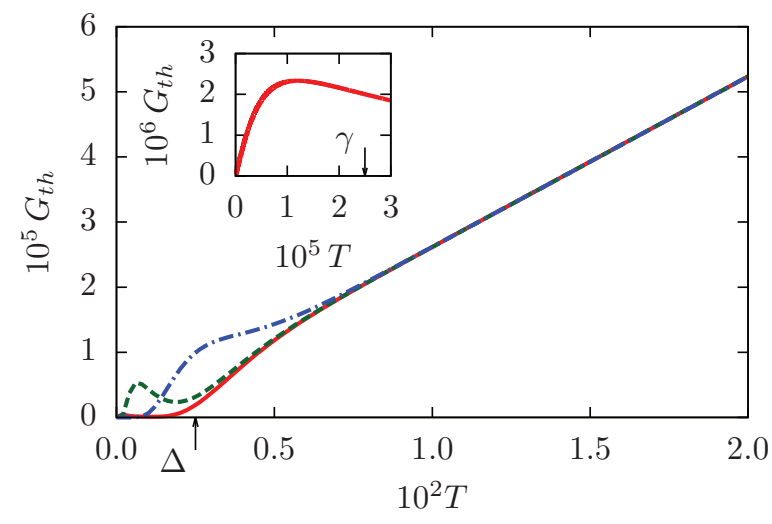

FIG. 4. (Color online) Low-temperature behavior of the tunneling thermal conductance through reservoirs 1 and 2 as a function of temperature, for different values of the chemical potential of the reservoirs: $\mu=0$ corresponding to resonance (red, solid), and two off-resonant values $\mu=\pi /(5 L)$ (green, dashed), $\pi / L$ (blue, dotdashed). The couplings are $w_{1}=w_{2}=0.1$, and the ring length is $L=400$. The arrows indicate $T=\gamma, \Delta$. A zoom of the linear regime for very low temperatures $(T \sim \gamma)$ in the resonant case is shown in the inset.

To summarize, the universal behavior of the thermal conductance, ${ }^{23}$

$$
G_{\mathrm{th}}^{\mathrm{c}}=\kappa_{0} T,
$$

is expected in the limit of ultralow temperature $k_{B} T \ll \gamma$ and for a resonant $\mu$. In the high-temperature regime $k_{B} T \gg \Delta$, the conductance becomes independent of $\mu$ and grows again linearly, but the slope is not universal and depends on the coupling constants as described by Eqs. (29) and (30).

\section{Capacitive coupling}

In this case, the heat current is given by Eq. (23), which depends on a difference of Bose-Einstein distribution functions. The detailed behavior depends on the spectral properties described by the transmission function $\mathcal{T}_{12}^{\mathrm{c}}(\omega)$ at low frequency $\omega$. As shown in the lower panel of Fig. 2(a), this function

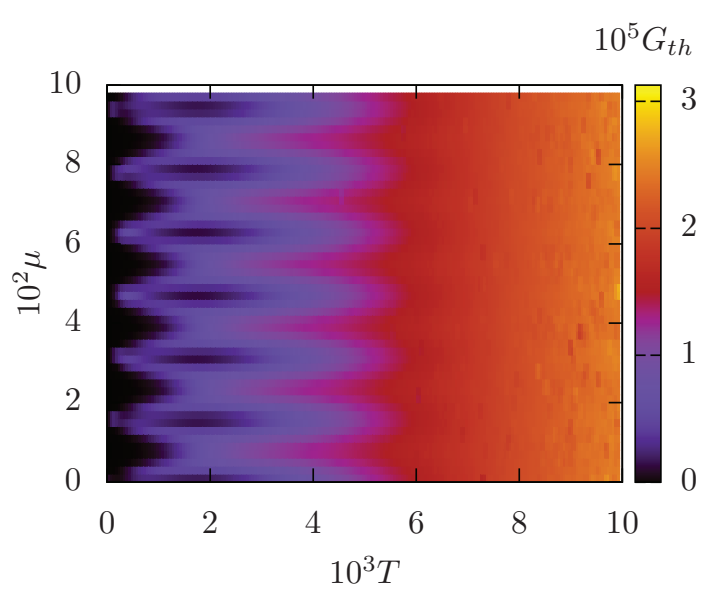

FIG. 5. (Color online) Map plot of the tunneling thermal conductance through reservoirs 1 and 2 , as function of the temperature and the chemical potential of the reservoirs. The couplings are $w_{1}=w_{2}=0.1$, and the ring length $L=400$. 
consists in a set of Lorentzian peaks centered at the energies of the uncoupled ring. Thus, for low enough temperatures, smaller than the mean level spacing $\Delta=2 \pi \hbar v_{F} / L,\left(k_{B} T<\right.$ $\Delta$ ), we can perform an expansion of the transmission function around $\omega=0$. It results in the frequency dependence

$$
\mathcal{T}_{12}^{\mathrm{c}}(\omega) \sim \gamma_{c} \omega^{2} \text {. }
$$

The low-temperature behavior for the heat current is then described by

$$
J^{Q, \mathrm{c}}=\gamma_{c} \int_{-\infty}^{+\infty} d \omega \omega^{3}\left[n\left(T_{1}\right)-n\left(T_{2}\right)\right] .
$$

The above integral can be evaluated in the limit of $T \rightarrow 0$ following standard procedures, ${ }^{29}$ leading to the result

$$
J^{Q, \mathrm{c}}=\left(\gamma_{c} k_{B}^{4} \pi^{2} / 30\right)\left(T_{1}^{4}-T_{2}^{4}\right),
$$

which implies the following law for the low-temperature behavior of the thermal conductance:

$$
G_{\text {th }}^{\mathrm{c}}(T)=\kappa_{1} T^{3}, \quad k_{B} T \ll \Delta,
$$

with

$$
\kappa_{1}=\kappa_{0} k_{B}^{2} \lambda_{1}, \quad \lambda_{1}=\frac{128 \pi^{6}}{5} \frac{\hbar^{2} v_{F}^{2}}{L^{2}}\left(\tilde{V}_{1} \tilde{V}_{2}\right)^{2},
$$

and $\tilde{V}_{\alpha}=\sqrt{\nu v_{\alpha}} V_{\alpha} /\left(\hbar v_{F}\right)\left(\hbar v_{F}^{\alpha}\right)$. For higher temperatures $\left(k_{B} T \gg \Delta\right)$, the analysis is more subtle. An analytical computation can be performed by considering an approximate form of (23), valid in this macroscopic regime. We describe this approach in Appendix D. In contrast to the tunneling case, here an intermediate regime may emerge provided that the coupling strengths $V_{1}$ and $V_{2}$ satisfy $1 / \sqrt{\tilde{V}_{1} \tilde{V}_{2}} \gg \Delta$. Under this condition, we can distinguish a regime where the thermal conductance follows again a cubic power law, but with a prefactor that is independent of the length of the edge,

$$
G_{\text {th }}^{\mathrm{c}}(T)=\kappa_{2} T^{3}, \quad \Delta \ll k_{B} T \ll 1 / \sqrt{\tilde{V}_{1} \tilde{V}_{2}},
$$

with

$$
\kappa_{2}=\kappa_{0} k_{B}^{2} \lambda_{2}, \quad \lambda_{2}=\frac{32 \pi^{4}}{5} \frac{\tilde{V}_{1}^{2} \tilde{V}_{2}^{2}}{\tilde{V}_{1}^{2}+\tilde{V}_{2}^{2}} .
$$

On the other hand, in the high-temperature regime defined by $k_{B} T \gg \frac{1}{\sqrt{\tilde{V}_{1} \tilde{V}_{2}}}$, the conductance reaches a saturation value,

$$
G_{\mathrm{th}}^{\mathrm{c}}=\frac{\sqrt{2} k_{B}}{\pi \hbar} \frac{\sqrt{\tilde{V}_{1} \tilde{V}_{2}}}{\tilde{V}_{1}^{2}+\tilde{V}_{2}^{2}}, \quad k_{B} T \gg \frac{1}{\sqrt{\tilde{V}_{1} \tilde{V}_{2}}} .
$$

In Fig. 3(b), we show the thermal conductance at a fixed temperature $T$ within the regime $k_{B} T \gg \Delta$ as function of the coupling strength. As in the case of tunneling contacts, the conductance decreases as the coupling goes to zero and as the coupling goes to infinity, while it peaks in between. This suggests a similar underlying mechanism to explain this behavior. The nature of the contact is, however, different and the coupling mismatching is in this case quantified by the parameter $\mathcal{M}_{\alpha}=\left(k_{B} T\right) \tilde{V}_{\alpha}$. A dimensional analysis indicates that $\left[V_{\alpha}\right]=E L^{2}$. Then, it is appropriate to recast this parameter as $V_{\alpha}=\mathcal{V}_{\alpha} /\left(k_{F}^{\alpha} k_{F}\right)$, where $\left[\mathcal{V}_{\alpha}\right]=E$ and $k_{F}^{\alpha}$ and $k_{F}$ are the Fermi wave vectors for particles with the Fermi energy within the reservoirs $\alpha$ and the edge, respectively.

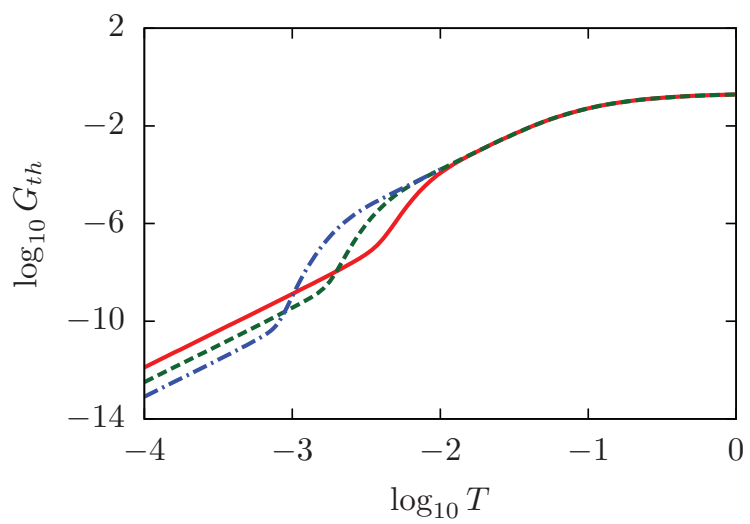

FIG. 6. (Color online) Capacitive thermal conductance as a function of the temperature for different ring lengths: $L=100$ (red, solid), 200 (green, dashed), and 400 (blue, dot-dashed). The couplings are $V_{1}=V_{2}=1$.

With these definitions $\tilde{V}_{\alpha}=\sqrt{\nu \nu_{\alpha}} \mathcal{V}_{\alpha} /\left(\varepsilon_{F}^{\alpha} \varepsilon_{F}\right)$, where $\varepsilon_{F}^{\alpha}$ and $\varepsilon_{F}$ are the Fermi energy of the electrons within the reservoirs and the edge, respectively. Thus $\left[\tilde{V}_{\alpha}\right]=E^{-1}$ and the mismatching measurement $\mathcal{M}_{\alpha}=\left(k_{B} T\right) \sqrt{\nu v_{\alpha}} \mathcal{V}_{\alpha} /\left(\varepsilon_{F}^{\alpha} \varepsilon_{F}\right)$ is dimensionless and can be interpreted as a ratio between the thermal energy times the coupling energy at the contact and the energy of the particles within the reservoir times the energy of the particles within the edge. As the temperature enters the matching measurement, the optimal coupling for which the conductance achieves its maximum value depends on $T$, as shown in Fig. 3(b). This behavior contrasts to the one of the thermal conductance for tunneling coupling, in which case the maximum is independent of $T$ [see Fig. 3(a)]. The dependence on $T$ of the matching measurement also suggests that the conductance saturates at high temperature. In fact, notice that in order to satisfy the quantum limit $G_{\mathrm{th}}^{\mathrm{c}} \leqslant \kappa_{0} T,{ }^{31}$ the following condition must be fulfilled $\lambda_{2}\left(k_{B} T\right)^{2} \leqslant 1$, which implies a constant value of $G_{\text {th }}^{\mathrm{c}}$ for $\left(k_{B} T\right)^{2}>1 /\left(\tilde{V}_{1} \tilde{V}_{2}\right)$ as shown in Eq. (39).

The behavior of the thermal conductance within the different regimes discussed in the present section are illustrated in Fig. 6 for systems with different lengths. Notice that the length of the system affects only the low-temperature cubic regime $k_{B} T<\Delta$. A final remarkable feature worth of notice is the dependence on the filling factors $v_{\alpha}$ and $v$ of the thermal conductance within the three regimes.

\section{B. Local temperature}

\section{Tunneling coupling}

In order to define the local temperature, we follow a procedure similar to that originally introduced in Ref. 30, which was also the one adopted in Ref. 15. We define a thermometer consisting in a third reservoir, which is noninvasively locally coupled to the edge at a point $x_{3}$, i.e., $w_{3} \rightarrow 0$. The latter reservoir is assumed to satisfy the condition of local thermal equilibrium with the edge, which means that it has a temperature $T_{3}$ for which the heat current $J_{3}^{Q, \mathrm{t}}=0$. The so determined temperature $T_{3}$, precisely, defines the local temperature sensed by the thermometer. We focus on the limit of low temperatures, compared to the chemical potential $\mu$ but 
within the range $T \gg \Delta$. The calculation is analogous to that of the thermal conductance:

$$
T_{3}=\left\{\begin{array}{lll}
\sqrt{A^{2} T_{1}^{2}+B^{2} T_{2}^{2}} & \text { if } & x_{1}<x_{2}<x_{3}, \\
\sqrt{C^{2} T_{1}^{2}+D^{2} T_{2}^{2}} & \text { if } & x_{1}<x_{3}<x_{2},
\end{array}\right.
$$

where the coefficients $A, B, C, D$ are given by

$$
\begin{aligned}
& A=\frac{\tilde{w}_{1}\left(1-\tilde{w}_{2}^{2}\right)}{\sqrt{\left(1+\tilde{w}_{1}^{2} \tilde{w}_{2}^{2}\right)\left(\tilde{w}_{1}^{2}+\tilde{w}_{2}^{2}\right)}}, \\
& B=\frac{\left(1+\tilde{w}_{1}^{2}\right) \tilde{w}_{2}}{\sqrt{\left(1+\tilde{w}_{1}^{2} \tilde{w}_{2}^{2}\right)\left(\tilde{w}_{1}^{2}+\tilde{w}_{2}^{2}\right)}}, \\
& C=\frac{\tilde{w}_{1}\left(1+\tilde{w}_{2}^{2}\right)}{\sqrt{\left(1+\tilde{w}_{1}^{2} \tilde{w}_{2}^{2}\right)\left(\tilde{w}_{1}^{2}+\tilde{w}_{2}^{2}\right)}}, \\
& D=\frac{\left(1-\tilde{w}_{1}^{2}\right) \tilde{w}_{2}}{\sqrt{\left(1+\tilde{w}_{1}^{2} \tilde{w}_{2}^{2}\right)\left(\tilde{w}_{1}^{2}+\tilde{w}_{2}^{2}\right)}},
\end{aligned}
$$

where again, $\tilde{w}_{\alpha}=w_{\alpha} / 2 \hbar \sqrt{v_{F} v_{F}^{\alpha}}$.

The results are shown in Fig. 7(a) for the choice of coupling parameters $w_{1}=w_{2}$ between the ring and the source and drain reservoirs, which are kept at the same chemical potential but different temperatures $T_{1}>T_{2}$. In Fig. 7(a), the profile for the local temperature as a function of the position $x_{3}$ at which the thermometer is connected is shown. The salient features of the figure are the discontinuities at the positions where the source and drain reservoirs are connected, which increase as the strength of the couplings to the source and drain reservoirs increase. Such a behavior is completely equivalent to that obtained in Ref. 15, where heat transport was induced by injection of an ac current at the source reservoir, instead of establishing an explicit temperature gradient.

The emergent picture is the following. Hot electrons tunnel from the source reservoir and propagate along the edge with a definite chirality along a given arm of the edge until they reach the colder drain reservoir, to which they can tunnel. Cold electrons tunnel from the drain reservoir and propagate with a given chirality along the other arm of the edge until they reach the drain reservoir, to which they can tunnel. The net result is the downstream arm of the edge mainly visited by hot electrons, while the upstream arm is visited by colder ones. The consequence is a higher temperature for the first arm of the edge in comparison to the second one, as observed in the Fig. 7(a). The temperature along the arms is approximately constant, with small finite size oscillations of $\mathcal{O}(1 / L)$, which are related to the structure of levels spaced in $\Delta \propto 1 / L$. The average values of these temperatures within each arm increases with the amplitude of the coupling to the reservoirs $w_{1}, w_{2}$.

\section{Capacitive coupling}

In analogy to the tunneling case, we define a thermometer as a third weakly coupled reservoir. In the present case, we assume a capacitive coupling $V_{3} \rightarrow 0$ between the edge and the thermometer. As in the previous case, we define the local temperature as the temperature $T_{3}$ of the third reservoir for which there is no heat current between this system and the edge, i.e., $J_{3}^{Q \text {,c }}=0$. The corresponding temperature

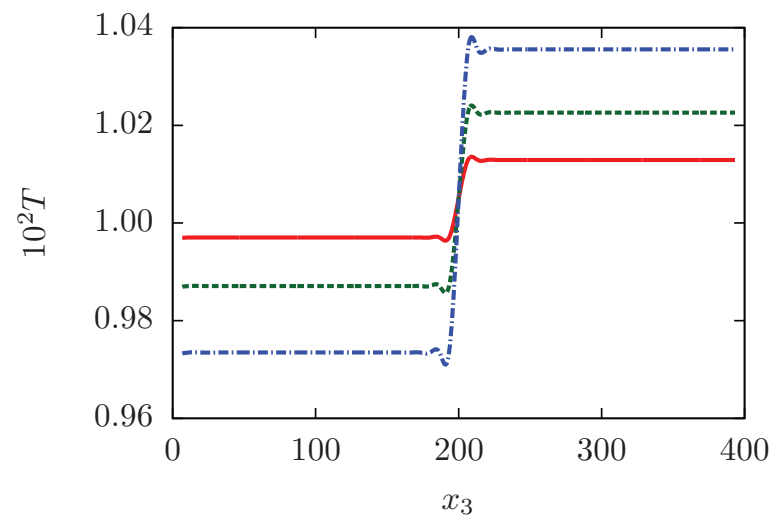

(a) Tunneling coupling

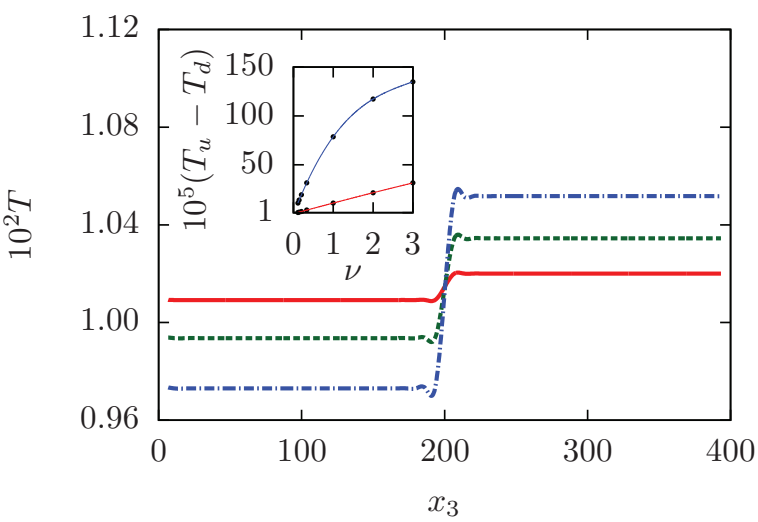

(b) Capacitive coupling

FIG. 7. (Color online) Local temperature along the edge, as a function of the position of the thermometer, for different values of the couplings with the reservoirs. For the tunneling case (a), we have set $w_{1}=w_{2}=0.4$ (red, solid), 0.6 (green, dashed), 0.8 (blue, dotted-dashed) and $\mu=0$. For the capacitive case (b), the values are $V_{1}=V_{2}=1$ (red, solid), 2 (green, dashed), and 3 (blue, dotteddashed). The remaining parameters are $x_{1}=0, x_{2}=200, L=400$, $T_{1}=0.009$, and $T_{2}=0.011$. A filling factor $v=1$ is considered. The temperature difference between the two arms of the ring $\left(T_{u}-T_{d}\right)$ as a function of $v$ is shown in the inset, for two values of the couplings: $V_{1}=V_{2}=1,3$.

profiles are shown in Fig. 7(b). In comparison to the plots for tunneling coupling shown in Fig. 7(a), we find the same qualitative behavior for the local temperature along the edge. Namely, discontinuities at the positions where the reservoirs are coupled and thermalization within each arm with the upstream reservoir, in agreement with the chiral propagation of the particles along the edge state. Concerning the effect of the filling factor on the temperature profile, its role is similar to a renormalization of the strength of the coupling to the reservoirs $V_{1}, V_{2}$, as can be inferred from the dependence of the Green functions on $v$ [see Eq. (B7)]. Thus the effect of thermalization of each branch with the closest upstream reservoir is more pronounced as this parameter increases. This is illustrated in the inset of Fig. 7(b), where we show the difference between the average temperature along the downstream branch $T_{u}$ and the corresponding one to the upstream one $T_{d}$, as a function of $v$ for two different values of the couplings to the reservoirs. The temperature jump $T_{u}-T_{d}$ increases with $v$ following a nonuniversal law. 


\section{SUMMARY AND CONCLUSIONS}

We have analyzed the heat transport through edge states of a two-dimensional electron gas in the quantum Hall effect. We considered two exactly solvable configurations. One of the cases corresponds to a system with filling factor $v=1$ coupled to reservoirs at different temperatures through tunneling couplings. The second case corresponds to capacitive coupling between the edge and the reservoirs and integer or fractional filling factor of the form $v=1 / m$.

The main features can be characterized in terms of two temperature regimes, defined with respect to the level spacing of the edge, $\Delta=\frac{2 \pi \hbar v_{F}}{L}$. In the mesoscopic regime, $T \ll \Delta$, finite size effects related to the discrete level spacing of the edge affect the behavior of the heat transport. In the tunneling case, the two terminal thermal conductance $G_{\text {th }}^{\mathrm{t}}$ has a different behavior depending on the position of the chemical potential, relative to the positions of the energy levels of the edge. For ultralow temperatures, $T \ll \gamma$, where $\gamma$ is the width of the peaks, it grows linearly with $T$, obeying the universal law $G_{\text {th }}^{\mathrm{t}}=\kappa_{0} T$, with $\kappa_{0}$ the universal thermal conductance quantum.

In the capacitive case, the thermal conductance behaves in a very different way within this regime. It displays a highly nonuniversal cubic law, $G_{\text {th }}^{\mathrm{c}} \propto T^{3}$, with a coefficient depending on the couplings to the reservoirs and the edge length, through the combination $\frac{V_{1}^{2} V_{2}^{2}}{L^{2}}$.

In the macroscopic regime, which takes place at higher temperatures, $T>\Delta$, finite-size effects become irrelevant, and thermal transport does not depend on the length of the system. However, the regime is not universal, in the sense that there is a strong dependence on the couplings to the reservoirs, for both tunneling and capacitive contacts. In particular, in the tunneling case, the two terminal thermal conductance is still a linear function of temperature, but with a proportionality coefficient that is a nonmonotonic function of the coupling strengths [see Eq. (30)]. In the capacitive case, this regime extends to any other filling $v$. Interestingly, an additional intermediate regime appears in this case for $\Delta \ll k_{B} T \ll$ $\frac{1}{\sqrt{\tilde{V}_{1} \tilde{V}_{2}}}$, with a thermal conductance characterized again by a cubic power law behavior, $G_{\text {th }}^{\mathrm{c}}(T)=\kappa T^{3}$, with $\kappa$ depending on the filling factors $v, v_{1}, v_{2}$, and the couplings $V_{1}$ and $V_{2}$. As in the tunneling case, $\kappa$ is a nonmonotonic function of the couplings [see Eq. (38)]. Finally, at high temperatures, $k_{B} T \gg \frac{1}{\sqrt{\tilde{V}_{1} \tilde{V}_{2}}}$, the capacitive thermal conductance reaches a saturation value, $G_{\text {th }}^{\mathrm{c}} \sim \frac{1}{\sqrt{v}} \frac{\sqrt{\sqrt{\nu_{1} \nu_{2}} \tilde{V}_{1} \tilde{V}_{2}}}{\nu_{1} \tilde{V}_{1}^{2}+\nu_{2} \tilde{V}_{2}^{2}}$. This result strongly differs from the linear behavior of the tunneling thermal conductance in the same regime. Concerning the dependence on the filling factors, we are not able to exactly treat the case with $v \neq 1$ for tunneling contacts, in order to verify if the conductance is also independent of the filling. However, if we notice that the two terminal electrical conductance for pointlike tunneling contacts is $G=e^{2} / h$, independent of $v{ }^{19}$ it is likely that this independence also holds for the thermal conductance in the presence of tunneling couplings.

Our results indicate that the behavior of the local temperature along the edge, defined from the coupling to a thermometer, is qualitatively the same for tunneling and capacitive couplings. As in the case of the ac-driven edge considered in Refs. 9 and 15, the landscape of local temperature as a function of the position along the edge, is fully consistent with the chiral propagation of the energy along the edge state. This means that given a configuration of an edge in contact to reservoirs with different temperatures, each piece of the edge tends to thermalize with the closest upstream reservoir. Remarkably, this behavior does not depend on the nature of the contact. It is qualitatively the same for a tunneling contact, which injects particles that carry energy, and for capacitive contact where only energy is exchanged.

The practical outcome of our results is the fact that capacitive couplings are as suitable as tunneling ones for the study of heat along edge states. This opens the possibility for the study of hybrid setups including capacitive and tunneling connections. This could be particularly interesting in the studies of edge states of quantum Hall states with fillings $v=2 / 3$ and $5 / 2$ that have charged along with neutral modes, which are insensitive to a capacitive coupling. On the other hand, since our results indicate that thermal transport with capacitive contacts is sensitive to the values of $v, v_{1}$, and $v_{2}$, one could conceive a capacitive thermal device designed to measure filling fractions of quantum Hall samples.

\section{ACKNOWLEDGMENTS}

We thank G. Lozano for discussions. We acknowledge support from CONICET, ANPCyT, UBACYT, UNLP (Argentina). EF thanks Programa Raíces (MINCYT, Argentina) for support and the Department of Physics, FCEyN UBA (Argentina) for hospitality. This work was supported in part by the National Science Foundation, under grant DMR-1064319 (EF).

\section{APPENDIX A: DYSON EQUATIONS FOR THE FERMIONIC GREEN FUNCTIONS-TUNNELING COUPLING}

We define the mixed retarded Green function,

$$
\tilde{G}^{R}\left(x, r_{\alpha} ; t, t^{\prime}\right)=-i \Theta\left(t-t^{\prime}\right)\left\langle\left\{\Psi(x, t), \Psi^{\dagger}\left(r_{\alpha}, t^{\prime}\right)\right\}\right\rangle,
$$

and the retarded Green function of the ring:

$$
G^{R}\left(x, x^{\prime} ; t, t^{\prime}\right)=-i \Theta\left(t-t^{\prime}\right)\left\langle\left\{\Psi(x, t), \Psi^{\dagger}\left(x^{\prime}, t^{\prime}\right)\right\}\right\rangle .
$$

The Dyson equations for these functions cast

$$
\begin{aligned}
& \left(\partial_{t^{\prime}}-v_{F} \partial_{x^{\prime}}\right) G^{R}\left(x, x^{\prime} ; t-t^{\prime}\right) \\
& =\delta\left(t-t^{\prime}\right) \delta\left(x-x^{\prime}\right)+\sum_{\alpha} w_{\alpha} \tilde{G}^{R}\left(x, r_{\alpha} ; t-t^{\prime}\right) \delta\left(r_{\alpha}-r_{\alpha}^{0}\right) \\
& \quad \times \delta\left(x-x_{\alpha}\right), \\
& \left(\partial_{t^{\prime}}-v_{F}^{\alpha} \partial_{r_{\alpha}}\right) \tilde{G}^{R}\left(x, r_{\alpha} ; t-t^{\prime}\right) \\
& =w_{\alpha} G^{R}\left(x, x^{\prime} ; t-t^{\prime}\right) \delta\left(r_{\alpha}-r_{\alpha}^{0}\right) \delta\left(x^{\prime}-x_{\alpha}\right) .
\end{aligned}
$$

We introduce the inverse of the differential operators,

$$
\begin{aligned}
& \left(\partial_{t^{\prime}}-v_{F} \partial_{x^{\prime}}\right) G^{0, R}\left(x-x^{\prime} ; t-t^{\prime}\right)=\delta\left(t-t^{\prime}\right) \delta\left(x-x^{\prime}\right), \\
& \left(\partial_{t^{\prime}}-v_{F}^{\alpha} \partial_{r_{\alpha}}\right) g^{R}\left(r_{\alpha}-r_{\alpha}^{\prime}, t-t^{\prime}\right)=\delta\left(t-t^{\prime}\right) \delta\left(r_{\alpha}-r_{\alpha}^{\prime}\right),
\end{aligned}
$$

which we identify as the free retarded Green functions of the ring and the reservoirs, respectively. Performing the Fourier 
transform with respect to $t-t^{\prime}$ and $r_{\alpha}-r_{\alpha}^{\prime}$ in Eqs. (A3a) and (A4), we obtain

$$
\begin{aligned}
G^{R}\left(x, x^{\prime} ; \omega\right)= & G^{0, R}\left(x, x^{\prime} ; \omega\right)-\sum_{p_{\alpha}} \tilde{G}^{R}\left(x, p_{\alpha} ; \omega\right) \\
& \times w_{p_{\alpha}} G^{0, R}\left(x_{\alpha}, x^{\prime} ; \omega\right), \\
\tilde{G}^{R}\left(x, p_{\alpha} ; \omega\right)= & -G^{R}\left(x, x_{\alpha} ; \omega\right) w_{p_{\alpha}} g^{R}\left(p_{\alpha}, \omega\right),
\end{aligned}
$$

where $w_{p_{\alpha}}=w_{\alpha} e^{-i p_{\alpha} r_{\alpha}^{0}} / \sqrt{L_{\alpha}}$ and

$$
g^{R}\left(p_{\alpha}, \omega\right)=\left[g^{A}\left(p_{\alpha}, \omega\right)\right]^{*}=\frac{1}{\omega-\varepsilon_{p_{\alpha}}+i \eta},
$$

with $\varepsilon_{p_{\alpha}}=v_{F}^{\alpha} p_{\alpha}$. Equation (A6b) can be replaced in Eq. (A6a) obtaining

$$
\begin{aligned}
G^{R}\left(x, x^{\prime} ; \omega\right)= & G^{0, R}\left(x, x^{\prime} ; \omega\right)+\sum_{\alpha=1}^{M} G^{R}\left(x, x_{\alpha} ; \omega\right) \Sigma_{\alpha}^{t, R}(\omega) \\
& \times G^{0, R}\left(x_{\alpha}, x^{\prime} ; \omega\right),
\end{aligned}
$$

where we have defined the retarded self-energies

$$
\Sigma_{\alpha}^{t, R}(\omega)=\left|w_{\alpha}\right|^{2} \int \frac{d p_{\alpha}}{2 \pi} g^{R}\left(p_{\alpha}, \omega\right),
$$

which enclose the effect of the coupling to the reservoir. It is useful to define the spectral function

$$
\Gamma_{\alpha}^{t}(\omega)=-2 \operatorname{Im}\left[\Sigma_{\alpha}^{t, R}(\omega)\right],
$$

which explicitly reads

$$
\Gamma_{\alpha}^{t}(\omega)=\frac{\left|w_{\alpha}\right|^{2}}{v_{F}^{\alpha}} \Theta(\Lambda-|\omega|),
$$

where $\Lambda$ is a high-energy cutoff that defines the bandwidth of the reservoir, while the corresponding explicit expression for the retarded self-energy is

$$
\Sigma_{\alpha}^{t, R}(\omega)=\ln \left|\frac{\Lambda-\omega}{\Lambda+\omega}\right|-i \frac{\Gamma_{\alpha}^{t}(\omega)}{2} .
$$

It is easy to verify that the Green functions evaluated from Eq. (A8) satisfy the following identity:

$$
\begin{aligned}
& G^{R}\left(x, x^{\prime} ; \omega\right)-G^{A}\left(x^{\prime}, x ; \omega\right) \\
& \quad=-i \sum_{\beta=1}^{M} G^{R}\left(x, x_{\beta} ; \omega\right) \Gamma_{\beta}^{t}(\omega) G^{A}\left(x_{\beta}, x^{\prime} ; \omega\right),
\end{aligned}
$$

being $G^{A}\left(x^{\prime}, x ; \omega\right)=\left[G^{R}\left(x, x^{\prime} ; \omega\right)\right]^{*}$ the advanced Green function.

In order to calculate the current, we need the lesser mixed Green function. The Dyson's equations can be straightforwardly derived from Eq. (A8) using Langreth's rules: ${ }^{32}$

$$
\begin{aligned}
\tilde{G}^{<}\left(x, p_{\alpha} ; \omega\right)= & -G^{<}\left(x, x_{\alpha} ; \omega\right) w_{p_{\alpha}} g^{A}\left(p_{\alpha}, \omega\right) \\
& -G^{R}\left(x, x_{\alpha} ; \omega\right) w_{p_{\alpha}} g^{<}\left(p_{\alpha}, \omega\right), \\
G^{<}\left(x, x^{\prime} ; \omega\right)= & \sum_{\alpha=1}^{M} G^{R}\left(x, x_{\alpha} ; \omega\right) \Sigma_{\alpha}^{t,<}(\omega)\left[G^{R}\left(x^{\prime}, x_{\alpha} ; \omega\right)\right]^{*}
\end{aligned}
$$

being

$$
\Sigma_{\alpha}^{t,<}(\omega)=i \int \frac{d \omega}{2 \pi} f_{\alpha}(\omega) \Gamma_{\alpha}^{t}(\omega),
$$

while

$$
g_{p_{\alpha}}^{<}(\omega)=2 i \pi f_{\alpha}(\omega) \delta\left(\omega-\varepsilon\left(p_{\alpha}\right)\right)
$$

with

$$
f_{\alpha}(\omega)=\frac{1}{e^{(\omega-\mu) / T_{\alpha}}+1}
$$

being the Fermi-Dirac distribution.

The Green function of the decoupled ring is given by the sum

$$
G^{0, R}\left(x, x^{\prime} ; \omega\right)=\frac{1}{L} \sum_{n=-N}^{N} \frac{e^{i k_{n}\left(x-x^{\prime}\right)}}{\omega-\varepsilon_{k_{n}}+i \eta},
$$

with $\varepsilon_{k}=v_{F} k$, being $k_{n}=2 n \pi / L$ and $N$ is a positive integer that defines the high-energy cutoff $\Lambda_{r}=v_{F} 2 \pi N / L$. In the limit of $N \rightarrow \infty$, the sum in the above noninteracting Green function can be computed analytically, ${ }^{33}$ and the result is

$$
\begin{aligned}
G^{0, R}\left(x, x^{\prime} ; \omega\right)= & \frac{1}{2 v_{F}} \frac{e^{-i \omega\left(x-x^{\prime}\right) / v_{F}}}{\sin \left(\omega L / 2 v_{F}\right)}\left[\Theta\left(x-x^{\prime}\right) e^{i \omega L /\left(2 v_{F}\right)}\right. \\
& \left.+\Theta\left(x^{\prime}-x\right) e^{-i \omega L /\left(2 v_{F}\right)}\right] .
\end{aligned}
$$

This last expression has been extensively used in all the analytical calculations.

\section{APPENDIX B: DYSON'S EQUATION FOR THE BOSONIC GREEN FUNCTION-CAPACITIVE COUPLING}

We follow a similar procedure to that exposed in the previous appendix. The differences are just due to the different type of commutation relations of Eq. (8) obeyed by the chiral bosonic fields. As in the fermionic case, we start by defining the retarded Green function corresponding to the ring,

$$
D^{R}\left(x, x^{\prime} ; t, t^{\prime}\right)=-i \Theta\left(t-t^{\prime}\right)\left\langle\left[\phi(x, t), \phi\left(x^{\prime}, t^{\prime}\right)\right]\right\rangle,
$$

and mixed degrees of freedom,

$$
\tilde{D}^{R}\left(x, r_{\alpha} ; t, t^{\prime}\right)=-i \Theta\left(t-t^{\prime}\right)\left\langle\left[\phi(x, t), \phi\left(r_{\alpha}, t^{\prime}\right)\right]\right\rangle .
$$

We derive the Dyson's equations by evaluating the time evolution of the fields. For the retarded Green functions defined above, we get

$$
\begin{aligned}
& \frac{1}{v}\left(\partial_{t^{\prime}}-v_{F} \partial_{x^{\prime}}\right) D^{R}\left(x, x^{\prime} ; t-t^{\prime}\right) \\
& \quad=\pi \delta\left(t-t^{\prime}\right) \operatorname{sgn}\left(x-x^{\prime}\right) \\
& \quad+2 \pi \sum_{\alpha} V_{\alpha} \partial_{r_{\alpha}} \tilde{D}^{R}\left(x, r_{\alpha} ; t-t^{\prime}\right) \delta\left(r_{\alpha}-r_{\alpha}^{0}\right) \delta\left(x-x_{\alpha}\right),
\end{aligned}
$$

$$
\begin{aligned}
& \frac{1}{v_{\alpha}}\left(\partial_{t^{\prime}}-v_{F}^{\alpha} \partial_{r_{\alpha}}\right) \tilde{D}^{R}\left(x, r_{\alpha} ; t-t^{\prime}\right) \\
& \quad=2 \pi V_{\alpha} \partial_{x^{\prime}} D^{R}\left(x, x^{\prime} ; t-t^{\prime}\right) \delta\left(r_{\alpha}-r_{\alpha}^{0}\right) \delta\left(x^{\prime}-x_{\alpha}\right) .
\end{aligned}
$$


It is now convenient to introduce the inverse of the operators $\left(\partial_{t^{\prime}}-v_{F}^{\alpha} \partial_{r_{\alpha}^{\prime}}\right)$ and $\left(\partial_{t^{\prime}}-v_{F}^{\alpha} \partial_{x^{\prime}}\right)$, which correspond to the solutions of the following partial differential equations

$$
\frac{1}{v_{\alpha}}\left(\partial_{t^{\prime}}-v_{F}^{\alpha} \partial_{r_{\alpha}^{\prime}}\right) d^{0, R}\left(r_{\alpha}, r_{\alpha}^{\prime} ; t-t^{\prime}\right)=\delta\left(t-t^{\prime}\right) \delta\left(r_{\alpha}-r_{\alpha}^{\prime}\right),
$$

$$
\frac{1}{v}\left\{\partial_{t^{\prime}}-v_{F} \partial_{x^{\prime}}\right\} D^{0, R}\left(x, x^{\prime} ; t-t^{\prime}\right)=\delta\left(t-t^{\prime}\right) \delta\left(x-x^{\prime}\right) .
$$

The Fourier transforms of these functions read

$$
\begin{gathered}
d^{0, R}\left(r_{\alpha}, r_{\alpha}^{\prime} ; \omega\right)=v_{\alpha} \int \frac{d p_{\alpha}}{2 \pi} \frac{e^{-i p_{\alpha}\left(r_{\alpha}-r_{\alpha}^{\prime}\right)}}{i\left(\omega-v_{F}^{\alpha} p_{\alpha}+i \eta\right)}, \\
D^{0, R}\left(x, x^{\prime} ; \omega\right)=\frac{v}{L} \sum_{n=-N}^{N} \frac{e^{i k_{n}\left(x-x^{\prime}\right)}}{\omega-\varepsilon_{k_{n}}+i \eta} .
\end{gathered}
$$

Fourier transforming with respect to $t-t^{\prime}$, combining Eqs. (B3a) and (B3b), and calculating the derivatives with respect to $x, x^{\prime}$ result in the expression

$$
\begin{aligned}
\mathcal{D}^{R}\left(x, x^{\prime} ; \omega\right)= & \mathcal{D}^{0, R}\left(x, x^{\prime} ; \omega\right)+\sum_{\alpha=1}^{M} \mathcal{D}^{R}\left(x, x_{\alpha} ; \omega\right) \Sigma_{\alpha}^{c, R}(\omega) \\
& \times \mathcal{D}^{0, R}\left(x_{\alpha}, x^{\prime} ; \omega\right),
\end{aligned}
$$

where

$$
\mathcal{D}^{R}\left(x, x^{\prime} ; \omega\right)=\frac{1}{2 \pi} \partial_{x} \partial_{x^{\prime}} D^{R}\left(x, x^{\prime} ; \omega\right)
$$

and

$$
\mathcal{D}^{0, R}\left(x, x^{\prime} ; \omega\right)=\partial_{x^{\prime}} D^{0, R}\left(x, x^{\prime} ; \omega\right) .
$$

Thus, as in the tunneling case, we have eliminated the degrees of freedom of the reservoirs from the Dyson's equation by defining the self-energies

$$
\begin{aligned}
\Sigma_{\alpha}^{c, R}(\omega)= & \left(2 \pi V_{\alpha}\right)^{2} \frac{v_{\alpha}}{v_{F}^{\alpha}} \lim _{r_{\alpha}^{\prime} \rightarrow r_{\alpha}^{0^{+}}} \partial_{r_{\alpha}^{\prime}} \\
& \times \int_{-P}^{+P} \frac{d p_{\alpha}}{2 \pi i} \frac{e^{-i p_{\alpha}\left(r_{\alpha}^{0}-r_{\alpha}^{\prime}\right)}}{\omega-v_{F}^{\alpha} p_{\alpha}+i \eta},
\end{aligned}
$$

which depend on the coupling to the reservoir as well as on their density of states. In the limit of the cutoff $\Lambda=v_{F} P \rightarrow$ $\infty$, we obtain

$$
\Sigma_{\alpha}^{c, R}=\frac{i}{2} \Gamma_{\alpha}^{c}(\omega)
$$

with

$$
\Gamma_{\alpha}^{c}(\omega)=(2 \pi)^{2} \frac{V_{\alpha}^{2}}{\left(v_{F}^{\alpha}\right)^{2}} v_{\alpha} \omega \Theta(\Lambda-|\omega|) .
$$

Notice that Eq. (B8) has the same structure as Eq. (A8). These Green functions, thus, satisfy the following identity, analogous to Eq. (A13):

$$
\begin{aligned}
& \mathcal{D}^{R}\left(x, x^{\prime} ; \omega\right)-\mathcal{D}^{A}\left(x^{\prime}, x ; \omega\right) \\
& \quad=-i \sum_{\alpha=1}^{M} \mathcal{D}^{R}\left(x, x_{\alpha} ; \omega\right) \Gamma_{\alpha}^{c}(\omega) \mathcal{D}^{A}\left(x_{\alpha}, x^{\prime} ; \omega\right),
\end{aligned}
$$

with $\mathcal{D}^{A}\left(x^{\prime}, x ; \omega\right)=\left[\mathcal{D}^{R}\left(x, x^{\prime} ; \omega\right)\right]^{*}$.
From Eq. (B8), we can derive the Dyson equations for the lesser Green functions by using the Langreth rules: ${ }^{32}$

$$
\mathcal{D}^{<}\left(x, x^{\prime} ; \omega\right)=\sum_{\alpha=1}^{M} \mathcal{D}^{R}\left(x, x_{\alpha} ; \omega\right) \Sigma_{\alpha}^{c,<}(\omega) \mathcal{D}^{A}\left(x_{\alpha}, x^{\prime} ; \omega\right),
$$

with

$$
\Sigma_{\alpha}^{c,<}(\omega)=i \Gamma_{\alpha}^{c}(\omega) n_{\alpha}(\omega)
$$

where

$$
n_{\alpha}(\omega)=\frac{1}{e^{\omega / T_{\alpha}}-1}
$$

is the Bose-Einstein distribution function corresponding to the temperature $T_{\alpha}$ of the reservoir.

Finally, using Langreth rules in the Fourier transform of Eq. (B3b) and performing the derivative with respect to $x$ and the second derivative with respect to $r_{\alpha}$, we obtain

$$
\begin{aligned}
\left.\partial_{x} \partial_{r_{\alpha}}^{2} D^{<}\left(x, r_{\alpha}^{\prime} ; \omega\right)\right|_{r_{\alpha}=r_{\alpha}^{0}} & \\
= & (2 \pi)^{2} V_{\alpha}\left[\left.\left.\mathcal{D}^{<}\left(x, x^{\prime} ; \omega\right)\right|_{x^{\prime}=x_{\alpha}} \partial_{r_{\alpha}}^{2} D^{0, A}\left(r_{\alpha}^{0}, r_{\alpha} ; \omega\right)\right|_{r_{\alpha}=r_{\alpha}^{0}}\right. \\
& \left.+\left.\left.\mathcal{D}^{R}\left(x, x^{\prime} ; \omega\right)\right|_{x_{\alpha}} \partial_{r_{\alpha}}^{2} D^{0,<}\left(r_{\alpha}^{0}, r_{\alpha} ; \omega\right)\right|_{r_{\alpha}=r_{\alpha}^{0}}\right],
\end{aligned}
$$

where $D^{0, A}\left(r_{\alpha}, r_{\alpha}^{\prime} ; \omega\right)=\left[D^{0, R}\left(r_{\alpha}^{\prime}, r_{\alpha} ; \omega\right)\right]^{*}$ and

$$
\left.\partial_{r_{\alpha}}^{2} D^{0,<}\left(r_{\alpha}^{0}, r_{\alpha} ; \omega\right)\right|_{r_{\alpha}=r_{\alpha}^{0}}=-\int_{-P}^{+P} d p_{\alpha} p_{\alpha}^{2} n_{\alpha}(\omega) \delta\left(\omega-v_{F}^{\alpha} p_{\alpha}\right) .
$$

\section{APPENDIX C: ANALYTIC CALCULATION OF THE THERMAL CONDUCTANCE FOR THE TUNNELING CASE IN THE MACROSCOPIC REGIME}

We evaluate the thermal conductance for the tunneling case, in the macroscopic regime. We will employ the method of contour integration. In the following, we set $v_{F}=v_{F}^{\alpha}=1$. Starting from Eq. (19), and taking into account that $T=\frac{1}{\beta} \gg$ $\Delta$, we find that $G_{\text {th }}^{t}$ is given by

$$
G_{\text {th }}^{t}=\int_{-\infty}^{\infty} \frac{d \omega}{2 \pi} \mathcal{T}_{12}^{t}(\omega) \frac{(\omega-\mu)^{2} \beta^{2} e^{\beta(\omega-\mu)}}{\left[1+e^{\beta(\omega-\mu)}\right]^{2}},
$$

with

$$
\mathcal{T}_{12}^{t}(\omega)=\Gamma_{1}(\omega) \Gamma_{2}(\omega)\left|G^{R}\left(x_{1}, x_{2} ; \omega\right)\right|^{2},
$$

where $\Gamma_{1}(\omega)$ is given by Eq. (A11), and $G^{R}\left(x_{1}, x_{2} ; \omega\right)$ is the full Green function of the ring.

In the limit $\Lambda \rightarrow \infty$, one has

$$
\begin{aligned}
\mathcal{T}_{12}^{t}(\omega)= & \frac{w_{1}^{2} w_{2}^{2}}{4\left(1+w_{1}^{2} w_{2}^{2} / 16\right)^{2}} \\
& \times \frac{1}{\sin ^{2}(\omega L / 2)+(\epsilon L / 2)^{2} \cos ^{2}(\omega L / 2)},
\end{aligned}
$$


where we defined

$$
\frac{\epsilon L}{2} \doteq \frac{\left(w_{1}^{2}+w_{2}^{2}\right)}{4\left(1+w_{1}^{2} w_{2}^{2} / 16\right)} .
$$

We evaluate the integral in Eq. (C1) by closing the contour over the upper half complex plane. The poles that lie inside the contour are located at the points

$$
\omega_{n}^{F}=\mu+\frac{(2 n+1) \pi i}{\beta}, \quad n=0,1,2, \ldots,
$$

and

$$
\omega_{n}=\frac{i}{L} \ln \left|\frac{1+\epsilon L / 2}{1-\epsilon L / 2}\right|+\frac{2 n \pi}{L} .
$$

The first series of poles does not contribute in the limit $T \gg \Delta$. The residues of the second series of poles are given by

$$
\begin{aligned}
\operatorname{Res}\left(\omega_{n}\right)= & \frac{-i}{2 \pi} \frac{w_{1}^{2} w_{2}^{2}}{4\left(1+w_{1}^{2} w_{2}^{2} / 16\right)^{2}} \frac{2}{L} \frac{\sqrt{1-(\epsilon L / 2)^{2}}}{\epsilon L / 2} \\
& \times \frac{\beta^{2}(2 n \pi / L-\mu)^{2} e^{\beta(2 n \pi / L-\mu)}}{\left[1+e^{\beta(2 n \pi / L-\mu)}\right]^{2}} .
\end{aligned}
$$

The integral can be written as a sum:

$$
G_{\mathrm{th}}^{t}=2 \pi i \sum_{n} \operatorname{Res}\left(\omega_{n}\right) .
$$

In the high-temperature limit $(\beta / L \rightarrow 0)$, we can transform the summation into an integral:

$G_{\mathrm{th}}^{t}=f\left(w_{1}, w_{2}\right) \frac{2 \pi}{L} \int_{-\infty}^{\infty} d n \frac{(2 n \beta \pi / L-\beta \mu)^{2} e^{\beta(2 n \pi / L-\mu)}}{\left[1+e^{\beta(2 n \pi / L-\mu)}\right]^{2}}$.

Performing the substitution $\frac{2 n \pi \beta}{L}-\beta \mu=k, d k=\frac{2 \pi \beta}{L} d n$ and evaluating the remaining integral, we obtain

$$
G_{\mathrm{th}}^{t}=f\left(w_{1}, w_{2}\right) \frac{\pi^{2}}{3} T,
$$

where

$$
f\left(w_{1}, w_{2}\right)=\frac{1}{2 \pi} \frac{w_{1}^{2} w_{2}^{2}}{w_{1}^{2}+w_{2}^{2}} \frac{1}{\left(1+w_{1}^{2} w_{2}^{2} / 16\right)} .
$$

The behavior displayed in Fig. 3(a) corresponds to the case $w_{1}=w_{2}=w$, which yields

$$
G_{\text {th }}^{t}=\frac{w^{2}}{4 \pi\left(1+w^{4} / 16\right)} \frac{\pi^{2}}{3} T .
$$

\section{APPENDIX D: ANALYTIC CALCULATION OF THE THERMAL CONDUCTANCE FOR THE CAPACITIVE CASE}

As in the tunneling case, we evaluate the thermal conductance by the method of contour integration. For simplicity, we set $v_{F}=v_{F}^{\alpha}=1$ and $v=v_{1}=v_{2}=1$. The dependence on these quantities will be recovered at the end of the computation. In the macroscopic regime, $T=\frac{1}{\beta} \gg \Delta$, the expression of Eq. (23) leads to

$$
G_{\mathrm{th}}^{c}=\int_{-\infty}^{\infty} \frac{d \omega}{2 \pi} \mathcal{T}_{12}^{c}(\omega) \frac{\omega^{2} \beta^{2} e^{\beta \omega}}{\left(1-e^{\beta \omega}\right)^{2}} .
$$

In the limit $\Lambda \rightarrow \infty$, the transmission coefficient is

$$
\begin{aligned}
\mathcal{T}_{12}^{c}(\omega)= & \frac{(2 \pi)^{4} V_{1}^{2} V_{2}^{2} \omega^{4}}{\left(1+\pi^{4} V_{1}^{2} V_{2}^{2} \omega^{4}\right)^{2}} \\
& \times \frac{1}{4\left[\sin ^{2}(\omega L / 2)+F^{2}(\omega) \cos ^{2}(\omega L / 2)\right]},
\end{aligned}
$$

where we have defined

$$
F(\omega)=\frac{\pi^{2}\left(V_{1}^{2}+V_{2}^{2}\right) \omega^{2}}{1+\pi^{4} V_{1}^{2} V_{2}^{2} \omega^{4}} .
$$

We evaluate the integral by closing the contour over the upper half complex plane. The poles that lie inside the contour are located at the points

$$
\omega_{n}^{B}=\frac{2 n \pi i}{\beta}, \quad n=1,2, \ldots,
$$

and at the points given by the solutions of the transcendental equation

$$
\tan \left(\frac{\omega L}{2}\right)=\frac{i \pi^{2}\left(V_{1}^{2}+V_{2}^{2}\right) \omega^{2}}{1+\pi^{4} V_{1}^{2} V_{2}^{2} \omega^{4}} .
$$

This equation cannot be solved exactly. However, the solutions can be very well approximated by

$$
\omega_{n} \approx \frac{2 n \pi}{L}+\frac{i}{L} \ln \left[\frac{1+F(2 n \pi / L)}{1-F(2 n \pi / L)}\right] .
$$

As in the capacitive case, the poles $\omega_{n}^{B}$ do not contribute to the integral in the limit $\beta / L \rightarrow 0$. Thus, the integral can be written in terms of the residues at $\omega=\omega_{n}$ as

$$
G_{\text {th }}^{c}=\frac{2 \pi}{L} \frac{V_{1}^{2} V_{2}^{2}}{\left(V_{1}^{2}+V_{2}^{2}\right)} \sum_{n} \frac{\omega_{n}^{4}}{\left(1+\pi^{4} V_{1}^{2} V_{2}^{2} \omega_{n}^{4}\right)} \frac{\beta^{2} e^{2 n \pi \beta / L}}{\left(1-e^{2 n \pi \beta / L}\right)^{2}} .
$$

In the limit $\beta / L \rightarrow 0$, we transform the summation into an integral and we then find

$$
G_{\mathrm{th}}^{c}=\frac{4}{\pi^{2}\left(V_{1}^{2}+V_{2}^{2}\right)} \int_{-\infty}^{\infty} d x \frac{\pi^{4} V_{1}^{2} V_{2}^{2} x^{4}}{\left(1+\pi^{4} V_{1}^{2} V_{2}^{2} x^{4}\right)} \frac{\beta^{2} e^{\beta x}}{\left(1-e^{\beta x}\right)^{2}},
$$

with $x=\frac{2 \pi n}{L}$. From this integral, we obtain the following limiting behaviors, within the macroscopic regime, $T=\frac{1}{\beta} \gg$ $\Delta$. At low temperatures, $T \sqrt{V_{1} V_{2}} \ll 1$, the conductance $G_{\text {th }}^{c}$ exhibits a power-law behavior,

$$
G_{\mathrm{th}}^{c}=\frac{16 \pi^{5}}{15} \frac{V_{1}^{2} V_{2}^{2}}{V_{1}^{2}+V_{2}^{2}} T^{3},
$$

whereas at high temperatures, $T \sqrt{V_{1} V_{2}} \gg 1$, it approaches a finite constant value,

$$
G_{\mathrm{th}}^{c}=\frac{\sqrt{2}}{\pi} \frac{\sqrt{V_{1} V_{2}}}{\left(V_{1}^{2}+V_{2}^{2}\right)} .
$$

The dependencies on the filling fractions and Fermi velocities are easily recovered by the substitutions $V_{\alpha} \rightarrow$ $\sqrt{\nu v_{\alpha}} V_{\alpha} / v_{F} v_{F}^{(\alpha)}$, with $\alpha=1,2$. 
${ }^{1}$ For a detailed discussion of edge states of quantum Hall systems see X.-G. Wen, Adv. Phys. 44, 405 (1995); A. M. Chang, Rev. Mod. Phys. 75, 1449 (2003); E. Fradkin, Field Theories of Condensed Matter Systems, 2nd ed. (Cambridge University Press, Cambridge, UK, 2013).

${ }^{2}$ R. B. Laughlin, Phys. Rev. B 23, 5632 (1981).

${ }^{3}$ B. I. Halperin, Phys. Rev. B 25, 2185 (1982).

${ }^{4}$ M. Büttiker, Phys. Rev. B 38, 9375 (1988).

${ }^{5}$ X. G. Wen and Q. Niu, Phys. Rev. B 41, 9377 (1990).

${ }^{6}$ X. G. Wen, Phys. Rev. Lett. 64, 2206 (1990).

${ }^{7}$ X. G. Wen, Phys. Rev. B 41, 12838 (1990).

${ }^{8}$ C. L. Kane and Matthew P. A. Fisher, Phys. Rev. Lett. 68, 1220 (1992); Phys. Rev. B 46, 15233 (1992); Phys. Rev. Lett. 72, 724 (1994).

${ }^{9}$ G. Granger, J. P. Eisenstein, and J. L. Reno, Phys. Rev. Lett. 102, 086803 (2009).

${ }^{10}$ A. Bid, N. Ofek, H. Inoue, M. Heiblum, C. L. Kane, V. Umansky, and D. Mahalu, Nature (London) 466, 585 (2010).

${ }^{11}$ C. Altimiras, H. le Sueur, U. Gennser, A. Cavanna, D. Mailly, and F. Pierre, Phys. Rev. Lett. 105, 226804 (2010).

${ }^{12}$ V. Venkatachalam, S. Hart, L. Pfeiffer, K. West, and A. Yacoby, Nat. Phys. 8, 676 (2012).

${ }^{13}$ C. Altimiras, H. le Sueur, U. Gennser, A. Anthore, A. Cavanna, D. Mailly, and F. Pierre, Phys. Rev. Lett. 109, 026803 (2012).

${ }^{14}$ I. Gurman, R. Sabo, M. Heiblum, V. Umansky, and D. Mahalu, Nat. Commun. 3, 1289 (2012).

${ }^{15}$ L. Arrachea and E. Fradkin, Phys. Rev. B 84, 235436 (2011).

${ }^{16}$ B. S. Kim, W. Zhou, Y. D. Yash, C. Zhou, N. Işik, and M. Grayson, Rev. Sci. Instrum. 83, 024703 (2012).
${ }^{17}$ D. T. McClure, Y. Zhang, B. Rosenow, E. M. Levenson-Falk, C. M. Marcus, L. N. Pfeiffer, and K. W. West, Phys. Rev. Lett. 103, 206806 (2009).

${ }^{18}$ C. de C. Chamon, D. E. Freed, and X. G. Wen, Phys. Rev. B 51, 2363 (1995).

${ }^{19}$ C. de C. Chamon and E. Fradkin, Phys. Rev. B 56, 2012 (1997).

${ }^{20}$ A. Cappelli, M. Huerta, and G. Zemba, Nucl. Phys. B 636, 568 (2002).

${ }^{21}$ Eytan Grosfeld and Sourin Das, Phys. Rev. Lett. 102, 106403 (2009).

${ }^{22}$ D. L. Kovrizhin and J. T. Chalker, Phys. Rev. Lett. 109, 106403 (2012).

${ }^{23}$ C. L. Kane and M. Fisher, Phys. Rev. 55, 15832 (1997).

${ }^{24}$ G. Viola, S. Das, E. Grosfeld, and A. Stern, Phys. Rev. Lett. 109, 146801 (2012)

${ }^{25}$ M. Stone, Bosonization (World Scientific, Singapore, 1994).

${ }^{26}$ F. D. M. Haldane, J. Phys. C 14, 2585 (1981).

${ }^{27}$ D. Karevski and T. Platini, Phys. Rev. Lett. 102, 207207 (2009).

${ }^{28}$ T. Prosen and B. Zuncovic, New J. Phys. 12, 025016 (2010).

${ }^{29}$ R. K. Pathria, Statistical Mechanics (Butterworth-Heinemann, Oxford, 1996).

${ }^{30}$ H. L. Engquist and P. W. Anderson, Phys. Rev. B 24, 1151 (1981).

${ }^{31}$ J. B. Pendry, J. Phys. A: Math. Gen. 16, 2161 (1983).

${ }^{32}$ See H. Haug and A. P. Jauho, Quantum Kinetics in Transport and Optics of Semiconductors, Springer Series in Solid-State Sciences Vol. 123 (Springer-Verlag, Berlin, Heidelberg, 1996).

${ }^{33}$ I. S. Gradshteyn and I. M. Ryzhik, Table of Integrals, Series and Products (Academic Press, New York, 1994). 